\title{
Water-Rock Interactions in Outer Solar System Bodies: Evidence from the Coordinated Analysis of Interplanetary Dust
}

\author{
Lindsay P. Keller ${ }^{1}$, Christopher Snead ${ }^{2}$, Scott Messenger ${ }^{1}$, George J. Flynn ${ }^{3}$, and Sue Wirick ${ }^{4}$ \\ 1. ARES, NASA-JSC, Houston, USA. \\ 2. Science Department, Jacobs, NASA-JSC, USA. \\ 3. SUNY-Plattsburgh, Plattsburgh, USA. \\ 4. Focused Beam Enterprises, Westhampton, USA.
}

NASA has an ongoing program of collecting interplanetary dust particles (IDPs) in the stratosphere using high altitude research aircraft. The collected IDPs are derived from asteroids and comets and here we report studies of a subset of hydrated IDPs rich in carbonaceous matter that are proposed to have a cometary origin [1]. Our studies are aimed at understanding the evolution of oxygen reservoirs in the Solar System and their interaction with cometary minerals and organic matter. The small size $(<20 \mu \mathrm{m})$ and fragility of these IDPs present a number of analytical challenges. We have pioneered techniques for performing chemical, mineralogical, isotopic, and spectroscopic measurements on the same sample in a carefully coordinated sequence.

Coordinated analyses of nanogram-size samples is made possible by several delicate sample preparation techniques. To avoid organic contamination, we embed IDPs in elemental sulfur and use ultramicrotomy to partly section the particles (the first few micrometers). Multiple thin sections (50-70 $\mathrm{nm}$ thick) are placed on different substrates depending on the analysis technique. We use a JEOL 2500SE scanning, transmission electron microscope (STEM) to determine the mineralogy, microstructure, and elemental compositions of constituent minerals in the thin sections through a combination of high resolution imaging, electron diffraction, quantitative energy-dispersive x-ray mapping, and electron energy-loss spectroscopy. Following the STEM analyses, we use a NanoSIMS 50L for high spatial resolution isotopic measurements of $\mathrm{H}, \mathrm{C}, \mathrm{N}$, and $\mathrm{O}$ to search for presolar grains and to understand the origin of the indigenous organic matter. The isotopic analyses are performed on the same sections analyzed in the STEM in order to correlate isotopic properties with the elemental and mineralogical data. We reserve other thin sections for non-destructive analyses utilizing synchrotronbased techniques including Fourier-transform infrared (FTIR) micro-spectroscopy and X-ray absorption near-edge structure (XANES) analyses, especially for functional group analysis of organic matter in the particles (Fig. 1). The remainder of the IDP is extracted from the sulfur bead that was used for microtomy and is pressed into $\mathrm{Au}$ foil for quantitative analysis (including light elements) using a JEOL 8530F field emission electron probe microanalyzer (EPMA). After the EPMA measurements, high precision oxygen isotopic analyses are obtained using Cameca IMS1270/1290 instruments at UCLA.

The hydrated IDPs in this study are dominated by saponitic clays, with minor magnetite, carbonate and abundant organic matter [2]. The remarkable oxygen isotopic compositions [3], high carbon contents, and the abundance of isotopically anomalous organic matter, together suggest that the high carbon, hydrated IDPs are derived from primitive sources not yet represented in meteorite collections such as outer main belt P- and D-type asteroids or comets. 
References:

[1] K. L. Thomas et al, Meteoritics 27 (1992), p. 296.

[2] L. P. Keller et al, Meteoritics Planet. Sci. (2016) \#6183.

[3] C. Snead et al, Lunar \& Planetary Science Conf. (2016) \#2850.

[4] K. Nakamura-Messenger et al, Science 314 (2006), p. 1439.

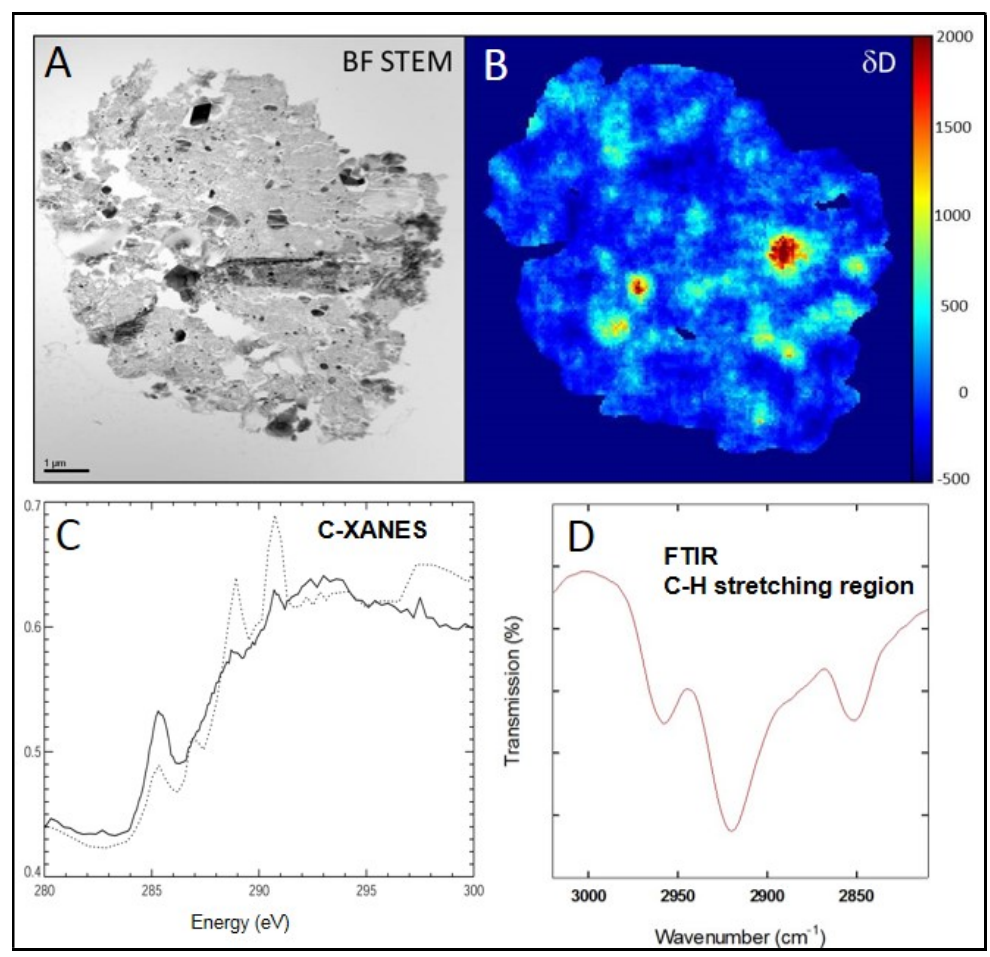

Figure 1. A) Brightfield STEM image of a thin section of C-rich hydrated IDP L2079C35 $\left(\Delta^{17} \mathrm{O}\right.$ $\sim 11 \%$ ) and B) its corresponding D/H image showing $\mathrm{D}$ hotspots that coincide with organic nanoglobules [4], C) C-XANES spectrum of the entire thin section (solid line) showing organic functional groups, dotted line is from a carbonate grain, and D) FTIR spectrum of the $\mathrm{C}-\mathrm{H}$ stretching region showing features consistent with the presence of shortchain aliphatic hydrocarbons.

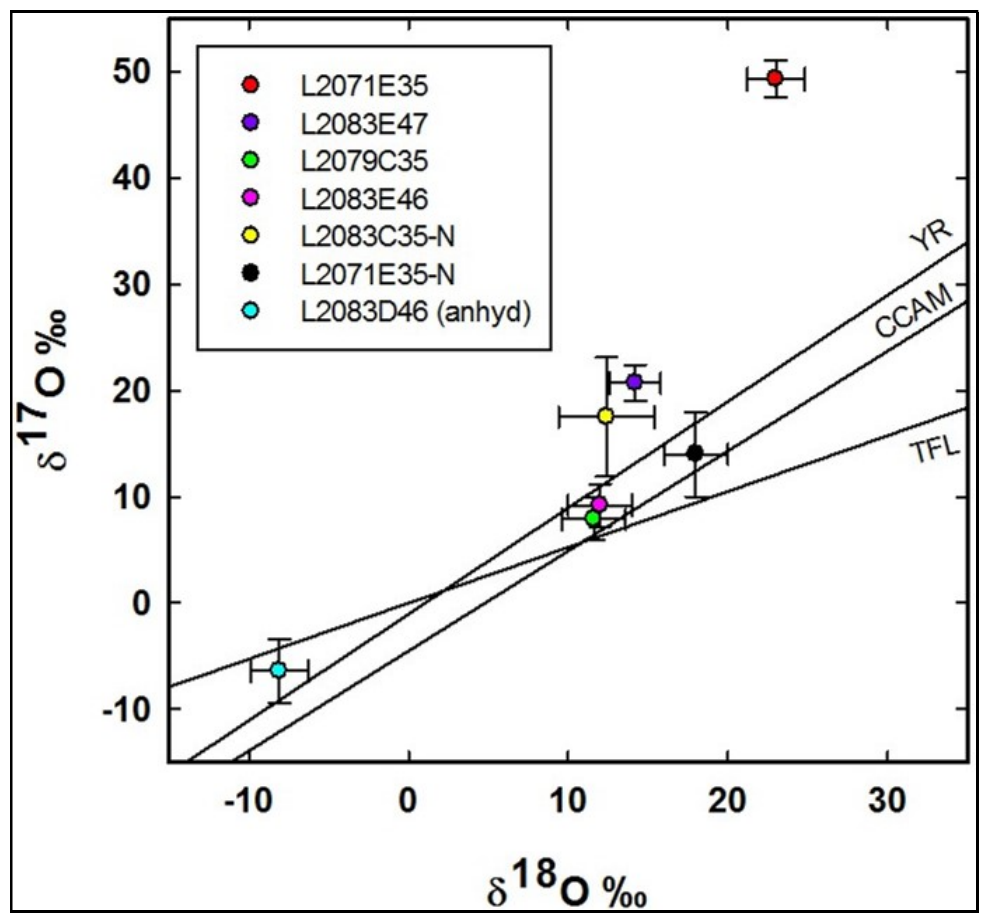

Figure 2. Oxygen isotope compositions of four hydrated IDPs and an anhydrous IDP ("D46"). Error bars are $2 \sigma$. NanoSIMS measurements from thin sections are indicated by ("-N") (adapted from [3]). 